\title{
ON TAME SUBGROUPS OF FINITELY PRESENTED GROUPS
}

\author{
RITA GITIK
}

\begin{abstract}
We prove that the free product of two finitely presented locally tame groups is locally tame and describe many examples of tame subgroups of finitely presented groups. We also include some open problems related to tame subgroups.
\end{abstract}

\section{INTRODUCTION}

A 3-manifold is called topologically tame if it is homeomorphic to the interior of a compact 3-manifold.

Marden's tameness conjecture [9] states that any hyperbolic 3-manifold with finitely generated fundamental group is topologically tame. The tameness conjecture became one of the central questions in the theory of hyperbolic 3-manifolds. The conjecture has been established by Agol in [1] and, independently, by Calegari and Gabai in [5]. Alternative proofs of the conjecture were given by Soma in [14] and Bowditch in [3].

The tameness conjecture is closely related to Simon's missing boundary manifold conjecture.

A 3-manifold $M$ is called a missing boundary manifold if it can be embedded in a compact manifold $\bar{M}$ such that $\bar{M}-M$ is a closed subset of the boundary of $\bar{M}$.

Simon conjectured in [13] that if $M_{0}$ is a compact orientable irreducible 3manifold, and $M$ is the cover of $M_{0}$ corresponding to a finitely generated subgroup of $\pi_{1}\left(M_{0}\right)$, then $M$ is a missing boundary manifold.

Long and Reid proved that Marden's conjecture implies Simon's conjecture for 3-manifolds which admit a geometric decomposition. Their proof appeared in [6].

The resolution of Thurston's Geometrization Conjecture by Perelman in 2003 showed that Simon's conjecture holds for all compact orientable irreducible 3manifolds. Accounts of Perelman's work were given by many mathematicians, see for example, [2] and [8.

Tucker proved in [15] that a non-compact orientable irreducible 3-manifold $M$ is a missing boundary manifold if and only if for any compact submanifold $C$ of $M$ the fundamental group of any connected component of $M-C$ is finitely generated. This observation made it possible to reduce the missing boundary manifold conjecture to a group-theoretic problem.

Mihalik introduced the notion of a 1-tame pair of groups in 10. His approach resulted in various group-theoretical results which implied some special cases of the tame ends conjecture, (cf. [10], [11]). The author introduced in [7] a different, but equivalent, definition of a tame subgroup.

Date: August 16, 2018.

2010 Mathematics Subject Classification. Primary: 20F65; Secondary: 20E06, 57M20, 57M10, $20 \mathrm{~F} 34$. 
Let $H$ be a subgroup of a group $G$. Choose the presentation $G=\langle X \mid R\rangle$. Let $T$ be the standard presentation 2 -complex of $G$, i.e. $T$ has one vertex, $T$ has an edge, which is a loop, for any generator $x \in X$, and $T$ has a 2-cell for any relator $r \in R$. The Cayley complex of $G$, denoted by Cayley $_{2}(G)$, is the universal cover of $T$. Denote by Cayley $_{2}(G, H)$ the cover of $T$ corresponding to a subgroup $H$ of $G$.

Definition 1. A finitely generated subgroup $H$ of a finitely presented group $G$ is tame in $G$ if for any finite subcomplex $C$ of $\mathrm{Cayle}_{2}(G, H)$ and for any component $K$ of Cayley ${ }_{2}(G, H)-C$ the group $\pi_{1}(K)$ is finitely generated.

The concept of a tame subgroup is of independent interest. For example, it is not known if there exists a finitely generated subgroup $H$ of a finitely presented group $G$ such that $H$ is not tame in $G$.

Mihalik demonstrated in [10] that Definition 1 is equivalent to the definition of a 1-tame pair $(G, H)$ given in [10. He also showed in [10] that $H$ is tame in $G$ if for one large finite subcomplex $C$ of Cayley $_{2}(G, H)$ the fundamental groups of the connected components of $\mathrm{Cayley}_{2}(G, H)-C$ are finitely generated. The complex $C$ can be chosen as a ball around the basepoint $H \cdot 1$.

The following resut was proved by Mihalik in [10].

Theorem 1. (Mihalik1) Let $X$ be a finite polyhedron with $\pi_{1}(X)=G$ and let $\tilde{X}$ be its universal cover. Let $H$ be a finitely generated subgroup of $G$ and let $H \backslash \tilde{X}$ be the quotient of $\tilde{X}$ by the action of $H$. Then $H$ is tame in $G$ if and only if for each finite subcomplex $C$ of $H \backslash \tilde{X}$ the fundamental group of every connected component of $((H \backslash \tilde{X})-C)$ is finitely generated.

Theorem (Mihalik1) implies that Definition 1 is independent of a finite presentation of the group $G$.

Following standard terminology, we introduce the following definition:

Definition 2. A group $G$ is locally tame if all finitely generated subgroups of $G$ are tame in $G$.

The main result of this paper is the following theorem.

Theorem 2. If $H$ and $K$ are finitely presented locally tame groups, then the free product $G=H * K$ is locally tame.

\section{Preliminaries}

Remark 1. It is not known if the trivial subgroup is tame in any finitely presented group. Moreover, it is not known if there exists an infinitely presented group such that the trivial subgroup is not tame in it.

Remark 2. The trivial subgroup is tame in any finitely generated abelian group (possibly with torsion) $A$. Indeed, $A$ is the fundamental group of a finite polyhedron $X$ of the form $\left(S^{1}\right)^{n} \times X_{m_{1}} \times \cdots \times X_{m_{k}}$, where $X_{m_{i}}$ is homeomorphic to a circle with a 2-disc attached by a degree $m_{i}$ map. The universal cover $\tilde{X}$ of $X$ is homeomorphic to $R^{n} \times M$, where $M$ is a compact set. Let $C$ be a compact subset of $\tilde{X}$. We want to show that all components of $\tilde{X}-C$ have finitely generated fundamental groups. We can replace $C$ by a larger compact set $K=p^{-1} p(C)$, where $p$ is the projection map from $R^{n} \times M$ onto $R^{n}$. The space $\left(R^{n} \times M\right)-K$ has components $\left(R^{n}-p(C)\right) \times M$, which have finitely generated fundamental groups. Hence Theorem (Mihalik1) implies the result. 
Remark 3. The trivial subgroup is tame in any negatively curved group, cf. [7] and 10].

Remark 4. If $H$ is a finite index subgroup of a finitely presented group $G$, then the trivial subgroup is tame in $H$ if and only if it is tame in $G$.

Indeed, let $X$ be a finite polyhedron with $\pi_{1}(X)=G$ and let $X_{H}$ be a finite cover of $X$ with $\pi_{1}\left(X_{H}\right)=H$. Note that the universal cover $\tilde{X}$ of $X$ is also a universal cover of $X_{H}$ and $X_{H}$ is a finite polyhedron. Hence Theorem (Mihalik1) implies the result.

Lemma 1. Let $G=K \times Z$, where $K$ is a locally tame finitely presented group. Let $t$ be a generator of $Z$ and let $H$ be a finitely presented subgroup of $G$ of the following form: $H=(H \cap K) \times t^{i}, i>0$. If $H \cap K$ is finitely generated, then $H$ is tame in $G$.

Proof. Let $Y$ be a finite polyhedron with $\pi_{1}(Y)=K$ and let $X=Y \times S^{1}$. Then $\pi_{1}(X)=G$.

Let $\tilde{X}$ be the universal covering space of $X$ and let $\tilde{Y}$ be the universal covering space of $Y$. Note that $\tilde{X}$ is homeomorphic to $\tilde{Y} \times R$. The quotient of $\tilde{X}$ by the action of $H$ is homeomorphic to $((H \cap K) \backslash \tilde{Y}) \times S^{1}$.

Let $C_{0}$ be a finite subcomplex of $((H \cap K) \backslash \tilde{Y}) \times S^{1}$.

Enlarge $C_{0}$ to a finite complex $C$ of the form $C=C_{1} \times S^{1}$, where $C_{1}$ is a finite subcomplex of $(H \cap K) \backslash \tilde{Y}$. Note that $((H \cap K) \backslash \tilde{Y}) \times S^{1}-C$ is homeomorphic to $\left(((H \cap K) \backslash \tilde{Y})-C_{1}\right) \times S^{1}$. By assumptions $H \cap K$ is finitely generated and $K$ is locally tame, hence the fundamental group of each component of $((H \cap K) \backslash \tilde{Y})-C_{1}$ is finitely generated, therefore the fundamental group of each component of

$\left(((H \cap K) \backslash \tilde{Y})-C_{1}\right) \times S^{1}$ is finitely generated. Hence Theorem (Mihalik1) implies that $H$ is tame in $G$.

Remark 5. Note that an infinitely generated subgroup might not be tame in a finitely presented group. For example, let $F=\langle a, b\rangle$ be a free group of rank 2 and let $H=\left\langle a^{n} b a^{-n}, n \in Z\right\rangle$ be a subgroup of $F$. As the fundamental group of the complement of any finite subcomplex of $\operatorname{Cayley}_{2}(F, H)$ is infinitely generated, it follows that $H$ is not tame in $F$.

Remark 6. Note that in general quasiisometry does not preserve tameness.

Let $A$ be a free abelian group of rank two. As was noted in Remark 2, the trivial subgroup is tame in $A$. The Cayley complex of $A$ is homeomorphic to the Euclidean plane. The Euclidean plane is quasiisometric to its subset $S$ consisting of the horizontal lines $\{y=n, n \in Z\}$ and the vertical lines $\{x=n, n \in Z\}$. The set $S$ is homeomorphic to the Cayley complex Cayley $\left(F, F^{\prime}\right)$, where $F$ is the free group of rank 2 and $F^{\prime}$ is its commutator subgroup.

Hence Cayley $\mathrm{C}_{2}(A)=\mathrm{Cayley}_{2}(A, 1)$ is quasiisometric to Cayley $\left(F, F^{\prime}\right)$. However, as the complement of any compact subset of $S$ in Cayley $2\left(F, F^{\prime}\right)$ has infinitely generated fundamental group, $F^{\prime}$ is not tame in $F$.

Remark 7. Let $G$ and $G_{0}$ be finitely presented groups, let $H$ be a finitely generated subgroup of $G$, and let $H_{0}$ be a finitely generated subgroup of $G_{0}$.

Assume that Cayle $y_{2}(G, H)$ is quasiisometric to Cayle $y_{2}\left(G_{0}, H_{0}\right)$.

It is not known if $H$ is tame in $G$ if and only if $H_{0}$ is tame in $G_{0}$. 
Remark 8. Let $G$ and $G_{0}$ be finitely presented quasiisometric groups. Let $H$ be a finitely generated subgroup of a group $G$ and let $H_{0}$ be a finitely generated subgroup of $G_{0}$.

Assume that $H$ is quasiisometric to $H_{0}$. It is not known if $H$ is tame in $G$ if and only if $H_{0}$ is tame in $G_{0}$.

This question is open even in the special case when $G=G_{0}$.

Remark 9. A finite group is locally tame, because its Cayley complex is finite.

Remark 10. A finite index subgroup $H$ of a finitely presented group $G$ is tame in $G$ because the complex Cayle $_{2}(G, H)$ is finite.

Remark 11. The trivial subgroup is tame in $Z$ because Cayley ${ }_{2}(Z)$ is homeomorphic to a straight line. As any non-trivial subgroup of $Z$ has finite index in $Z$, it follows that $Z$ is locally tame.

Remark 12. Free groups are locally tame. Indeed, for any free group $F$ and its finitely generated subgroup $H$ the complex $\mathrm{Cayle}_{2}(F, H)$ is one-dimensional. When $H$ is finitely generated, Cayle $y_{2}(F, H)$ is homotopic to a wedge of finitely many circles.

The author proved the following result in [7.

Theorem 3. (Gitik) Let $N$ be a finitely generated normal subgroup of a finitely generated group $G$. Then $N$ is tame in $G$ if the trivial subgroup is tame in the factor group $G / N$.

Theorem (Gitik) implies the following fact.

Lemma 2. A finitely generated abelian group is locally tame.

Proof. Any finitely generated abelian group $A$ is a direct product of the form $A=$ $Z^{n} \times$ Tor, where $Z^{n}$ is the direct product of $n$ copies of $Z$ and Tor is a finite abelian group. If $A$ is finite, it is locally tame by Remark 9 . If $A$ is infinite and $N$ is a subgroup of $A$, the factor group $A / N$ is a finitely generated abelian group. Remark 2 states that the trivial subgroup is tame in $A / N$, hence it follows from Theorem (Gitik) that $N$ is tame in $A$.

The following property is closely related to tameness.

Definition 3. A $C W$-complex $W$ has property $*$ if for any finite subcomplex $C$ the fundamental group of each component of $W-C$ is finitely generated.

Lemma 3. A $C W$-complex $W$ has property $*$ if and only if any finite cover of $W$ has property $*$.

Proof. Assume $W$ has property *. Let $U$ be a finite cover of $W$ and let $C$ be a finite subcomplex of $U$. Let $p: U \rightarrow W$ be the covering map. Then $p(C)$ is a finite subcomplex of $W$ and $C \subset p^{-1}(p(C))$. Each component of $U-p^{-1}(p(C))$ is a finite cover of a component of $W-C$, hence the fundamental group of each component of $U-p^{-1}(p(C))$ is a finite index subgroup of a component of $W-C$. As $W$ has property $*$, it follows that the fundamental group of each component of $U-p^{-1}(p(C))$ is finitely generated. However $U-C$ is obtained from $U-p^{-1}(p(C))$ by adding a finite complex, hence $U$ has property $*$.

Assume that $U$ has property $*$ and let $K$ be a finite subcomplex of $W$. Then $p^{-1}(K)$ is a finite subcomplex of $U$ and each component of $U-p^{-1}(K)$ has a 
finitely generated fundamental group. Each component of $W-U$ is finitely covered by a component of $U-p^{-1}(K)$, hence the fundamental group of each component of $U-p^{-1}(K)$ is a finite index subgroup of a component of $W-K$, hence $W$ has property $*$.

Mihalik proved the following result in [11].

Theorem 4. (Mihalik2) Let $1 \rightarrow A \rightarrow G \rightarrow B \rightarrow 1$ be a short exact sequence of infinite finitely presented groups, and let $H$ be a finitely generated subgroup of $A$ of infinite index in $A$. Then $H$ is tame in $G$.

Lemma 4. Let $G_{0}$ be a finite index subgroup of a finitely presented group $G$. Then a finitely generated subgroup $H$ of $G$ is tame in $G$ if and only if $H_{0}=H \cap G_{0}$ is tame in $G_{0}$.

Proof. Let $X$ be a $C W$-complex with $\pi_{1}(X)=G$ and let $X_{0}$ be a finite cover of $X$ with $\pi_{1}\left(X_{0}\right)=G_{0}$. Let $X_{H}$ be a cover of $X$ with $\pi_{1}(X)=H$ and let $X_{H_{0}}$ be a finite cover of $X_{H}$ with $\pi_{1}\left(X_{H_{0}}\right)=H_{0}$.

Consider the following commutative diagram of covering spaces, where the maps are covering projections.

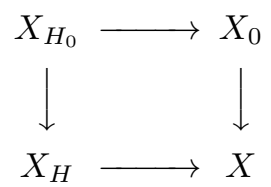

Assume that $H$ is tame in $G$. Theorem (Mihalik1) implies that $X_{H}$ has property *. Then Lemma 4 implies that $X_{H_{0}}$ has property $*$ and Theorem (Mihalik1) implies that $H_{0}$ is tame in $G_{0}$.

Assume that $H_{0}$ is tame in $G_{0}$. Theorem (Mihalik1) implies that $X_{H_{0}}$ has property $*$. Then Lemma 4 implies that $X_{H}$ has property $*$, and Theorem (Mihalik1) implies that $H$ is tame in $G$.

The author proved the following result in [7].

Lemma 5. (Gitik) Let $H$ be a subgroup of a group $G$, and let $H_{0}$ be a finite index subgroup of $H$. Then $H$ is tame in $G$ if and only if $H_{0}$ is.

Theorem (Mihalik2), Theorem (Gitik), and Lemma (Gitik) imply the following results.

Remark 13. Let $N$ be a normal finitely generated subgroup of a finitely presented group $G$.

(1) If $G / N$ is infinite, then any finitely generated subgroup of $N$ is tame in $G$, provided the trivial subgroup is tame in $G / N$.

Indeed, Lemma (Gitik) and Theorem (Gitik) imply the result for finiteindex subgroups of $N$, and Theorem (Mihalik2) implies the result for infiniteindex subgroups of $N$, provided $G / N$ is infinite.

(2) Lemma (Gitik) implies that if $G / N$ is finite, then any finitely generated subgroup of $N$ is tame in $G$ if and only if it is tame in $N$.

Lemma 6. Let $H$ and $K$ be infinite finitely presented groups, and let $G=H \times K$. Let $S$ be a finitely generated subgroup of $G$. If the trivial subgroup is tame in $H$ and in $K$, then $S \cap H$ and $S \cap K$ are tame in $G$. 
Proof. Consider the short exact sequence $1 \rightarrow H \rightarrow G \rightarrow K \rightarrow 1$. If $S \cap H$ has infinite index in $H$, the result follows from Theorem (Mihalik2). If $S \cap H$ has finite index in $H$, Lemma (Gitik) implies that $S \cap H$ is tame in $G$ if and only if $H$ is tame in $G$. Theorem (Gitik) implies that $H$ is tame in $G$ if the trivial subgroup is tame in $G / H=K$.

Lemma 7. Let $H$ and $K$ be finitely generated groups and let $G=H \times K$. If the trivial subgroup is tame in $H$ and in $K$ then it is tame in $G$.

Proof. Indeed, if $H$ and $K$ are infinite the result follows from Theorem (Mihalik2). If both $H$ and $K$ are finite, the result follows from Remark 9. If $H$ is finite and $K$ is infinite the result follows from Remark 4.

Remark 14. Let $G=H \times Z$. As the trivial subgroup is tame in $Z$, Theorem (Gitik) implies that $H$ is tame in $G$.

Let $H_{0}$ be a finitely presented subgroup of $H$. If $H_{0}$ is of infinite index in $H$, Theorem (Mihalik2) states that $H_{0}$ is tame in $G$. If $H_{0}$ is of finite index in $H$, Lemma (Gitik) states that $H_{0}$ is tame in $G$.

\section{Proof of Theorem 2}

We need the following notation.

Let $X^{*}=\left\{x, x^{-1} \mid x \in X\right\}$. For $x \in X$ define $\left(x^{-1}\right)^{-1}=x$.

Let $G$ be a group generated by a set $X$ and let $H$ be a subgroup of $G$. Let $\{H g\}$ be the set of right cosets of $H$ in $G$.

The coset graph of $G$ with respect to $H$, denoted Cayley $(G, H)$, is the oriented graph whose vertices are the cosets $\{H g\}$, the set of edges is $\{H g\} \times X^{*}$, and an edge $(H g, x)$ begins at the vertex $H g$ and ends at the vertex $H g x$. Denote the Cayley graph of $G$ by Cayley $(G)$. Note that $\operatorname{Cayley}(G, H)$ is the quotient of $\operatorname{Cayley}(G)$ by left multiplication by $H$. Also note that the 1-skeleton of $\operatorname{Cayley}_{2}(G)$ is $\operatorname{Cayley}(G)$, and the 1-skeleton of $\mathrm{Cayley}_{2}(G, H)$ is $\operatorname{Cayley}(G, H)$.

Let $G$ be generated by a disjoint union of sets $X$ and $Y$. We call a subset $C$ of Cayley $(G, H)$ an $X$-component, if all edges of $C$ have the form $(H g, x)$ with $x \in X^{*}$. We call $C$ a $Y$-component if all edges of $C$ have the form $(H g, y)$ with $y \in Y^{*}$.

Proof. Let $S$ be a finitely generated subgroup of $G=H * K$, where $H$ is generated by the set $X$ and $K$ is generated by the set $Y$. We assume that $X$ and $Y$ are disjoint.

The Kuros' subgroup theorem states that $S$ is a free product of a free group with subgroups of conjugates of $H$ or $K$. It follows that $S$ is the fundamental group of a graph of groups, where the vertex groups are subgroups of conjugates of $H$ or $K$ and the edge groups are trivial, cf. 12 .

Note that the complex Cayley $_{2}(G, S)$ has the following structure. All maximal $X$-components of Cayle $_{2}(G, S)$, except for a finite number, are homeomorphic to $\mathrm{Cayley}_{2}(H)$. The remaining finitely many maximal $X$-components of Cayle $_{2}(G, S)$ are homeomorphic to $\operatorname{Cayle}_{2}\left(H, H_{i}\right)$, where $H_{i}, 1 \leq i \leq n$ is a finitely generated subgroup of $H$. All maximal $Y$-components of $\mathrm{Cayle}_{2}(G, S)$, except for a finite number, are homeomorphic to $\mathrm{Cayle}_{2}(K)$. The remaining finitely many maximal $Y$-components of $\mathrm{Cayle}_{2}(G, S)$ are homeomorphic to Cayley $_{2}\left(K, K_{i}\right)$, where $K_{i}, 1 \leq i \leq n$ is a finitely generated subgroup of $K$. 
Let $C$ be a compact subset of $\operatorname{Cayley}_{2}(G, S)$. Then $C$ has non-empty intersection with only finitely many maximal $X$-components and maximal $Y$-components of Cayle $_{2}(G, S)$. As $H$ and $K$ are locally tame, the fundamental groups of each component of the complement of $C$ in any maximal $X$-component and any maximal $Y$ component is finitely generated. Hence the fundamental group of $\operatorname{Cayley}_{2}(G, S)-C$ is finitely generated.

\section{Open Questions}

(1) Let $G$ be a finitely presented group which is a direct product of subgroups $H$ and $K$, and let $S$ be a finitely presented subgroup of $G$ such that $S \cap H$ and $S \cap K$ are finitely generated. Is it true that $S$ is tame in $G$ if and only if $S \cap H$ is tame in $H$ and $S \cap K$ is tame in $K$ ?

(2) If $H$ is locally tame, does it follow that a cyclic extension of $H$ is locally tame?

A special case of this question: is $G=H \times Z$ locally tame?

Are finitely generated polycyclic groups, in particular, finitely generated nilpotent groups, locally tame?

(3) Is the trivial subgroup tame in the Thompson group $F$ (cf. [4]) with the infinite presentation $\left\langle x_{0}, x_{1}, x_{2}, \cdots \mid x_{k}^{-1} x_{n} x_{k}=x_{n+1}, k<n\right\rangle$ ?

Is the trivial subgroup tame in the Thompson group $F$ with the finite presentation $\langle A, B|\left[A B^{-1}, A^{-1} B A\right],\left[A B^{-1}, A^{-2} B A^{2}\right\rangle$ ?

(4) Let $N$ be a finitely generated normal subgroup of a finitely presented group $G$. Assume that $N$ and $G / N$ are locally tame and $N$ has fgip in $G$. Is $G$ locally tame?

Remark 15. Recall that a finitely generated subgroup $K$ has fgip in $G$ if the intersection of $K$ with any finitely generated subgroup of $G$ is finitely generated.

The following result would follow from an affirmative answer to Question 1.

Let $G, H$ and $K$ be as in Question 1. Assume that $H$ and $K$ are locally tame. If $H$ and $K$ have fgip in $G$, then $G$ is locally tame.

Indeed, let $S$ be a finitely generated subgroup of $G$. As $H$ and $K$ have fgip in $G$, the intersections $S \cap H$ and $S \cap K$ are finitely generated. As $H$ and $K$ are locally tame, it follows that $S \cap H$ is tame in $H$ and $S \cap K$ is tame in $K$. Hence the affirmative answer to Question 1 would imply that $S$ is tame in $G$. As $S$ is an arbitrary finitely generated subgroup of $G$, it follows that $G$ is locally tame.

\section{ACKNOWLEDGMENT}

The author wants to thank Peter Scott for helpful conversations.

\section{REFERENCES}

[1] I. Agol, Tameness of Hyperbolic 3-Manifolds,

[2] L. Bessieres, G. Besson, M. Boileau, S. Maillot, and J. Porti, Geometrization of 3-manifolds, EMS Tracts in Mathematics, 13(2010), European Mathematical Society, Zurich.

[3] B. Bowditch, Notes on Tameness, Enseign. Math., 56(2010), 229-285.

[4] J. W. Cannon and W. J. Floyd, WHAT IS...Thompson's Group?, Notices AMS, 58(2011), $1112-1113$

[5] D. Calegari and D. Gabai, Shrinkwrapping and the Taming of Hyperbolic 3-Manifolds, J. AMS, 19(2006), 385-446. 
[6] R. Canary, Marden's Tameness Conjecture: History and Applications, in Geometry, Analysis, and Topology of Discrete Groups, editors L. Ji, K. Liu, L. Yang, and S. T. Yau, Advanced Lectures in Mathematics, 6(2008), International Press, Sommerville MA, 137-162.

[7] R. Gitik, Tameness and Geodesic Cores of Subgroups, J. Austral. Math. Soc (Series A), 69(2000), 153-161.

[8] B. Kleiner and J. Lott, Notes on Perelman's papers, Geometry and Topology, 12(2008), pp. $2587-2858$.

[9] A. Marden, The Geometry of Finitely Generated Kleinian Groups, Annals of Math, 99 (1974), 383-462.

[10] M. Mihalik, Compactifying Coverings of 3-Manifolds, Comment. Math. Helv., 71(1996), 362372.

[11] M. Mihalik, Group Extensions and Tame Pairs, Trans. AMS, 351(1999), 1095-1107.

[12] G. P. Scott and C. T. C. Wall, Topological Methods in Group Theory, in Homological Group Theory, London Math. Soc. Lecture Notes Series, 36(1979), 137-214.

[13] J. Simon, Compactification of Covering Spaces of Compact 3-Manifolds, Mich. Math. J., 23(1976), 245-256.

[14] T. Soma, Existence of Ruled Wrappings in Hyperbolic 3-Manifolds, Geometry and Topology, 10 (2006), 1173-1184.

[15] T. W. Tucker, Non-Compact 3-Manifolds and the Missing Boundary Problem, Topology, 13(1974), 267-273.

E-mail address: ritagtk@umich.edu

Department of Mathematics, University of Michigan, Ann Arbor, Mi, 48109 